\title{
Self-Referenced Plasmon Waveguide Resonance Sensor Using Different Waveguide Modes
}

\author{
Pengfei Zhang, ${ }^{1,2}$ Le Liu, ${ }^{3}$ Yonghong He, ${ }^{1,2}$ Yanhong $\mathrm{Ji}^{4}$ and Hui $\mathrm{Ma}^{1,2}$ \\ ${ }^{1}$ Shenzhen Key Laboratory for Minimal Invasive Medical Technologies, Institute of Optical Imaging and Sensing, \\ Graduate School at Shenzhen, Tsinghua University, Shenzhen 518055, China \\ ${ }^{2}$ Department of Physics, Tsinghua University, Beijing 100084, China \\ ${ }^{3}$ Institute of Green Chemistry and Energy, Graduate School at Shenzhen, Tsinghua University, Shenzhen 518055, China \\ ${ }^{4}$ MOE Key Laboratory of Laser Life Science \& Institute of Laser Life Science, South China Normal University, \\ Guangzhou 510631, China \\ Correspondence should be addressed to Yonghong He; heyh@sz.tsinghua.edu.cn
}

Received 21 August 2014; Revised 30 October 2014; Accepted 6 November 2014

Academic Editor: Jae Ho Han

Copyright (c) 2015 Pengfei Zhang et al. This is an open access article distributed under the Creative Commons Attribution License, which permits unrestricted use, distribution, and reproduction in any medium, provided the original work is properly cited.

\begin{abstract}
We report a plasmon waveguide resonance (PWR) sensing structure supporting two waveguide modes for self-referenced measurement. We show theoretically the dual mode PWR sensing structure owns unique opportunities for self-referenced measurement, and the accuracy and cross sensitivity can be optimized by simply varying the gold film thickness or dielectric layer thickness or dielectric film refractive index. This structure may provide an approach owning good compatibility with the surface plasmon resonance and PWR biosensors for self-referenced biosensing.
\end{abstract}

\section{Introduction}

Surface plasmon is electromagnetic surface wave propagating along the interface of two materials with real dielectric constants of opposite signs. The surface plasmon resonance (SPR) is a sensing approach measuring the refractive index (RI) changes within the evanescent field of these waves [1]. The SPR has the advantage of high sensitivity and permits label free molecular interaction analysis in real time, which has become a central method for characterizing and quantifying biomolecular interactions [2-4]. The variations within the evanescent field can change the SPR propagation constant which results in a change in the sensor output, and these variations can be divided into the surface and bulk effects corresponding to the thickness change of the binding surface layer and bulk RI change, respectively [5]. In the practical applications, the surface effects usually need to be distinguished for specific detection, which is especially significant for the biosensing $[2,6]$. However, the typical SPR supporting only one surface plasmon mode cannot distinguish the surface and bulk effects. As a solution to this problem, the dual mode SPR sensors for self-referenced measurement are developed. The dual mode SPR sensors are usually based on the long range SPR sensing structure, which is constructed by sandwiching a dielectric layer between the prism and metal film in the SPR structure. The dual mode SPR sensors can simultaneously support the long and short range surface plasmon modes with different penetration depths, where the surface and bulk effects can be distinguished owing to the different sensitivity factors to the surface and bulk effect in different surface plasmon modes [7]. The optimal dual mode SPR sensors based on the wavelength interrogation [8], angular interrogation [9], and bimetallic film [10] were developed recently.

Plasmon waveguide resonance (PWR) is an extension of the SPR and is usually constructed by sequentially coating a dielectric layer on the metal surface in SPR sensors. The PWR sensors own high stability and enhanced evanescent field due to the shield and optical amplification effects of dielectric layer and permit the measurement in both transverse magnetic (TM) and transverse electric (TE) modes owing to the transformation of the admittance [11, 12]. But the PWR sensors were usually applied for bulk RI sensing due to the long 
penetration depth of the evanescent field [13]. To improve the PWR sensor performance in the surface sensing, recently the self-referenced PWR sensing method was proposed utilizing different penetration depths of the TM and TE modes [14]. This self-referenced PWR sensor has better performance than the SPR in the RI sensing. But compared with the dual mode SPR sensors, this sensor cannot allow the simultaneous measurement in different modes because the measurements in the TM and TE modes at one sensor spot need to be timemultiplexed.

In this paper we report a PWR structure using different waveguide modes with different penetration depths in TM mode for self-referenced measurement. We pay attention to the feasibility of the two waveguide modes' simultaneous excitation using a monochrome angular interrogation in this sensing film structure. In this case, one can use the conventional angular SPR sensors for self-reference. We show this sensing structure can be optimized for high accuracy of finding resonance position and low cross sensitivity between the surface and bulk responses. Besides, the sensor chip fabrications are also discussed for suitable materials to construct this sensor.

\section{Principle of the Self-Referenced PWR}

The key point of the self-referenced measurement is the different sensitivity factors for surface and bulk effects, which is corresponding to different penetration depths, in different modes. Inspired by different waveguide modes existence in the dielectric layer with different RI [15], we assume there will be different waveguide modes changing another parameter of the dielectric layer, namely, the thickness. In this study, we employ the typical PWR sensing structure shown in Figure 1(a), where a thin gold film of $50 \mathrm{~nm}$ and the silica film are sequentially coated on a BK7 prism. The water, a common material in nature, is employed as the sample and the incident wavelength is fixed at the $632.8 \mathrm{~nm}$. After increasing the thickness of dielectric layer to $1000 \mathrm{~nm}$, which is thicker than hundreds of nanometers in the typical PWR [11-13], we calculate the reflection angular spectrum and the electric field strength distribution using the Fresnel formula and Maxwell equations as shown in Figures 1(b) and 1(c), respectively. To show different penetration depths of TM1 and TM2 modes, the normalized electric field strength distribution in the water is shown in Figure 1(d). The refractive indices of the prism, gold, silica, and water used in the simulation are 1.515, 0.133 $+3.654 i, 1.46$, and 1.33 , respectively. The accuracy difference is because the silica film owns different silicon/oxygen ratio with different coating process so the reported $\mathrm{RI}$ is different $[16,17]$, and the water RI varies with the density at room temperature and fixed incident wavelength so it is not stable in different experiments [18]. So the accuracy of refractive indices of silica and water is lower than the optical glass prism and gold, whose refractive indices are usually stable with a given incident wavelength at room temperature [19].

It can be seen from Figures 1(b) and 1(c) that there are two resonance dips in the TM modes, namely, the TM1 and TM2 modes. The TM1 mode exists in the conventional PWR sensor. With the increasing thickness of the silica layer, the resonance angle, which is the incident angle affording the minimum reflectance intensity, in the TM1 mode shifts to the large value compared with the reported results in [13]. That may be caused by the increasing effective RI within the evanescent field due to the silica owning larger RI than the water. This can be also seen from the electric field distribution shown in Figure 1(c), where the penetration depth in the TM1 mode in the water is shortened because the electric field is mostly limited in the silica layer. Owing to these, the penetration depths in TM1 and TM2 modes are different in this sensing structure. This makes this sensor possible for selfreferenced measurement. Then we will discuss the surface and bulk sensitivity factors of this sensor based on the linear sensor response model [7]. The resonance angle shifts in the TM1 and TM2 modes, $\Delta \theta_{\mathrm{TM} 1}$ and $\Delta \theta_{\mathrm{TM} 2}$, are given by

$$
\begin{aligned}
& \Delta \theta_{\mathrm{TM} 1}=S_{\mathrm{S}-\mathrm{TM} 1} \Delta d+S_{\mathrm{B}-\mathrm{TM} 1} \Delta n, \\
& \Delta \theta_{\mathrm{TM} 2}=S_{\mathrm{S}-\mathrm{TM} 2} \Delta d+S_{\mathrm{B}-\mathrm{TM} 2} \Delta n,
\end{aligned}
$$

where the $S_{\mathrm{S} \text {-TM1 }}$ and $S_{\mathrm{S} \text {-TM2 }}$ are the surface sensitivity factors in degree-angle/nanometer-thickness for TM1 and TM2 modes and the $S_{\mathrm{B}-\mathrm{TM} 1}$ and $S_{\mathrm{B}-\mathrm{TM} 2}$ are the bulk sensitivities in degree-angle/refractive index unit (RIU) for TM1 and TM2 modes. The $\Delta d$ and $\Delta n$ are the binding layer thickness change in nanometer and the bulk RI change in RIU, respectively. In the experiment, if we know the sensitivity factors and resonance angle shifts, the surface layer thickness and bulk RI changes can be calculated as

$$
\begin{gathered}
\Delta d=\frac{\Delta \theta_{\mathrm{TM} 1} / S_{\mathrm{B}-\mathrm{TM} 1}-\Delta \theta_{\mathrm{TM} 2} / S_{\mathrm{B}-\mathrm{TM} 2}}{S_{\mathrm{S}-\mathrm{TM} 1} / S_{\mathrm{B}-\mathrm{TM} 1}-S_{\mathrm{S}-\mathrm{TM} 2} / S_{\mathrm{B}-\mathrm{TM} 2}}, \\
\Delta n=\frac{\Delta \theta_{\mathrm{TM} 1} / S_{\mathrm{S}-\mathrm{TM} 1}-\Delta \theta_{\mathrm{TM} 2} / S_{\mathrm{S}-\mathrm{TM} 2}}{S_{\mathrm{B}-\mathrm{TM} 1} / S_{\mathrm{S}-\mathrm{TM} 1}-S_{\mathrm{B}-\mathrm{TM} 2} / S_{\mathrm{S}-\mathrm{TM} 2}} .
\end{gathered}
$$

It can be seen that the $S_{\mathrm{S}-\mathrm{TM} 1}, S_{\mathrm{S}-\mathrm{TM} 2}, S_{\mathrm{B}-\mathrm{TM} 1}$, and $S_{\mathrm{B}-\mathrm{TM} 2}$ are the dominant performance indices in the self-referenced measurement. So the self-referenced sensor characteristic is usually evaluated with the difference in the sensitivity ratios as

$$
\chi=\left|\frac{S_{\mathrm{S}-\mathrm{TM} 1}}{S_{\mathrm{S}-\mathrm{TM} 2}}-\frac{S_{\mathrm{B}-\mathrm{TM} 1}}{S_{\mathrm{B}-\mathrm{TM} 2}}\right|,
$$

where the figure of merit $\chi$ is inversely proportional to the cross sensitivity [7]. The higher figure of merit $\chi$ indicates better ability to distinguish the surface and bulk effects. We suppose the RI of the binding layer is 1.48 (proteins) and calculate the sensitivity factors in two modes. The bulk and surface sensitivity factors can be calculated with

$$
S_{\mathrm{B}}=\frac{\partial \theta}{\partial n}, \quad S_{\mathrm{S}}=\frac{\partial \theta}{\partial d},
$$

where $\theta$ is the resonance angle, $n$ is the RI of sample, and $d$ is the thickness of binding layer [5]. The bulk sensitivity factors in TM1 and TM2 modes are calculated to be 6.6 and 


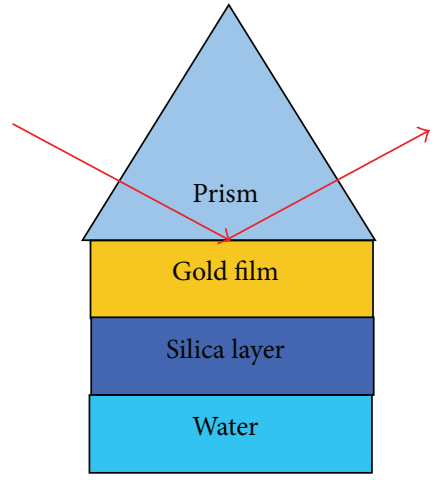

(a)

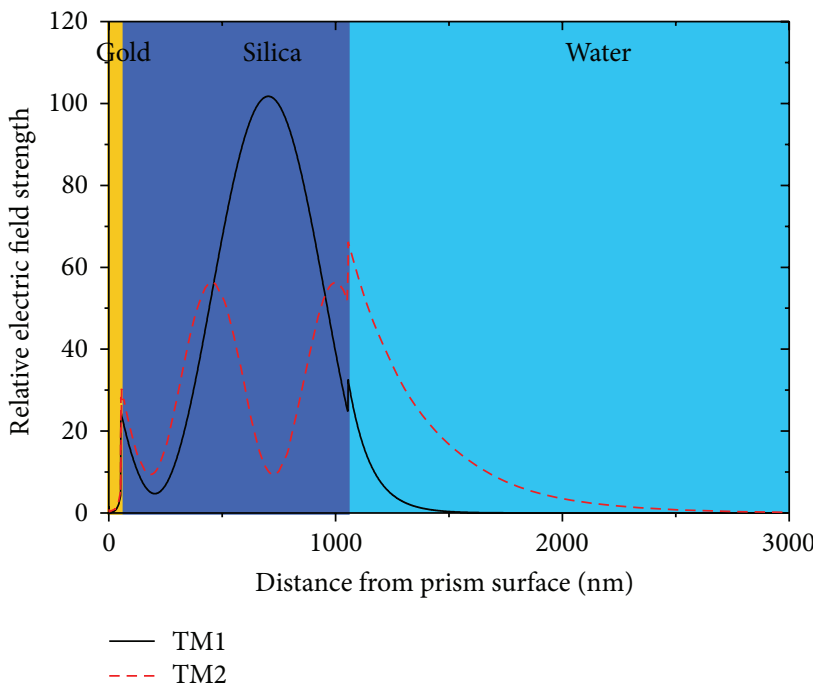

(c)

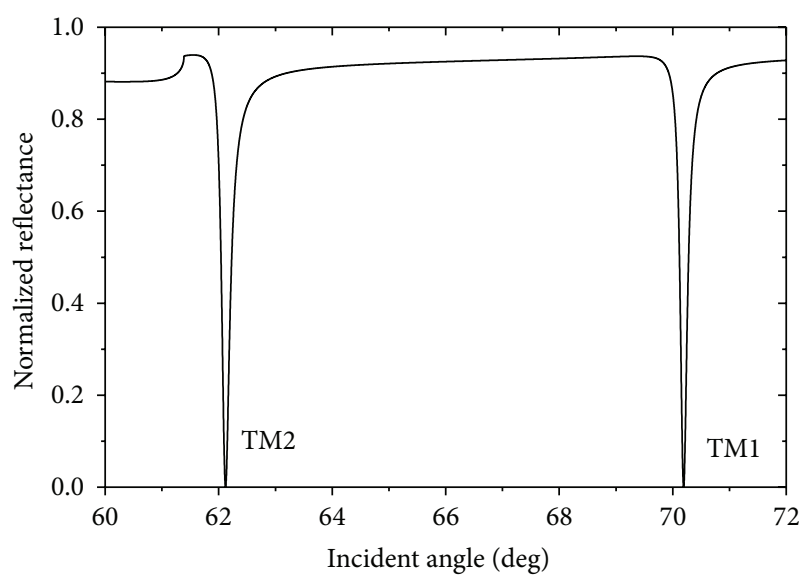

(b)

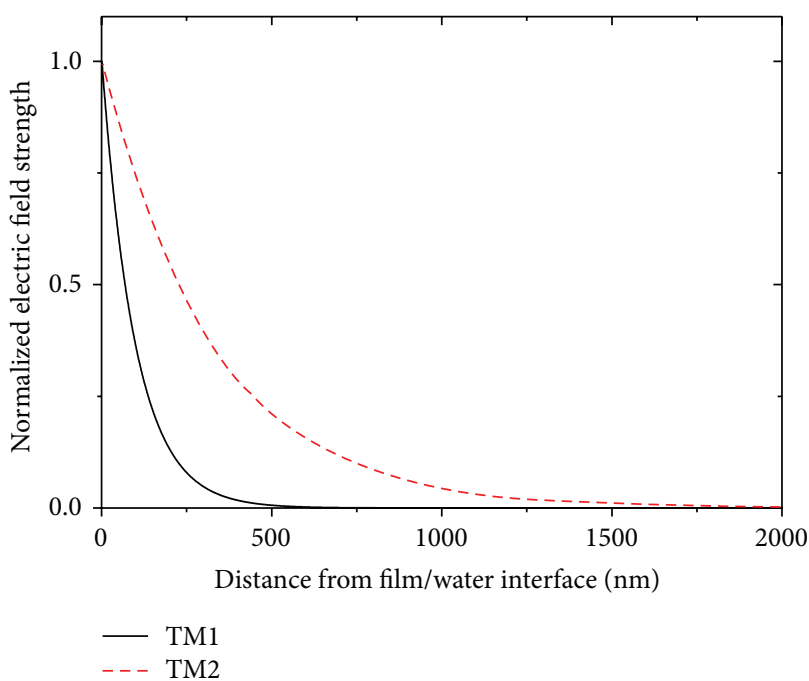

(d)

FIGURE 1: (a) The schematic of the plasmon waveguide resonance sensing structure. (b) The angular reflectance spectrum. The gold and silica films have the thickness of 50 and $1000 \mathrm{~nm}$, respectively. The refractive indices of the prism, gold, silica, and water are $1.515,0.133+3.654 i$, 1.46 , and 1.33, respectively. The incident wavelength is $632.8 \mathrm{~nm}$. (c) The electric field strengths in different waveguide modes under resonance condition are plotted against the distance from the prism surface. (d) The normalized electric field strengths in different waveguide modes under resonance conditions are plotted against the distance from the film/water interface.

39.2 degrees per RIU, and the surface sensitivity factors in TM1 and TM2 modes are calculated to be 0.0083 and 0.0144 degrees per nanometer, respectively. So the $\chi$ is approximately 4 in the simulation, which is comparable with the reported values [7-10]. Considering this, we believe that we propose a PWR sensing structure which is feasible for self-referenced measurement.

\section{Numerical Analysis of the Self-Referenced PWR}

The angular reflectance spectrum in the transverse magnetic (TM) mode can be calculated using the multiple reflectance theory and Fresnel formula with

$$
\begin{aligned}
& R_{p}=\left|\frac{r_{12}+r_{2 \cdots k} \exp \left(2 i \beta_{2}\right)}{1+r_{12} r_{2 \ldots k} \exp \left(2 i \beta_{2}\right)}\right|^{2}, \\
& r_{2 \cdots k}=\frac{r_{23}+r_{3 \cdots k} \exp \left(2 i \beta_{3}\right)}{1+r_{23} r_{3 \cdots k} \exp \left(2 i \beta_{3}\right)}, \\
& r_{i, j}=\frac{X_{i}-X_{j}}{X_{i}+X_{j}}, \quad X_{i}=\frac{\varepsilon_{i}}{k_{i z}}, \\
& k_{i z}=\frac{\omega}{c} \sqrt{\varepsilon_{i}-\varepsilon_{0} \sin ^{2} \theta},
\end{aligned}
$$

where $k$ is the total layer number, $\varepsilon_{j}$ and $d_{j}$ are the dielectric constant and thickness of the $j$ th layer, $\omega$ and $\theta$ are the angular 
frequency and incident angle of the incident light, and $c$ is the light speed in the vacuum, respectively $[20,21]$.

In this study, the electric field strength distribution in the self-referenced PWR sensing film structure can be calculated using the algorithm proposed by Hansen [22] and Tien and Ulrich [23]. Firstly, the field components of the electromagnetic wave propagating in each layer can be calculated with

$$
\begin{aligned}
& \left(\begin{array}{ll}
m_{11} & m_{12} \\
m_{21} & m_{22}
\end{array}\right)=\left(\begin{array}{cc}
\cos \left(\beta_{g}\right) & -\frac{\sin \left(\beta_{g}\right)}{p p_{g}} \\
-p p_{g} \times \sin \left(\beta_{g}\right) & \cos \left(\beta_{g}\right)
\end{array}\right) \\
& \cdot\left(\begin{array}{cc}
\cos \left(\beta_{d}\right) & -\frac{\sin \left(\beta_{d}\right)}{p p_{d}} \\
-p p_{l} \times \sin \left(\beta_{d}\right) & \cos \left(\beta_{d}\right)
\end{array}\right), \\
& \left(\begin{array}{c}
H y_{g} \\
E x_{g}
\end{array}\right)=\left(\begin{array}{cc}
\cos \left(k z_{g} \times z\right) & \frac{\sin \left(k z_{g} \times z\right)}{p p_{g}} \\
p p_{g} \times \sin \left(k z_{g} \times z\right) & \cos \left(k z_{g} \times z\right)
\end{array}\right) \\
& \cdot\left(\begin{array}{ll}
m_{11} & m_{12} \\
m_{21} & m_{22}
\end{array}\right) \cdot\left(\begin{array}{c}
t H \\
p p_{s} \times t H
\end{array}\right) \cdot n_{p} \cdot E i \\
& \left(\begin{array}{l}
H y_{d} \\
E x_{d}
\end{array}\right) \\
& =\left(\begin{array}{cc}
\cos \left(k z_{d}\left(z-d_{g}\right)\right) & \frac{\sin \left(k z_{d}\left(z-d_{g}\right)\right)}{p p_{l}} \\
p p_{d} \times \sin \left(k z_{d}\left(z-d_{g}\right)\right) & \cos \left(k z_{d} \times\left(z-d_{g}\right)\right)
\end{array}\right) \\
& \cdot\left(\begin{array}{cc}
\cos \left(\beta_{d}\right) & -\frac{\sin \left(\beta_{d}\right)}{p p_{d}} \\
-p p_{l} \times \sin \left(\beta_{d}\right) & \cos \left(\beta_{d}\right)
\end{array}\right) \\
& \cdot\left(\begin{array}{c}
t H \\
p p_{s} \times t H
\end{array}\right) \cdot n_{p} \cdot E i \\
& k z_{g}=\frac{2 \pi n_{g}}{\lambda} \cos \left(\theta_{g}\right), \quad k z_{d}=\frac{2 \pi n_{d}}{\lambda} \cos \left(\theta_{d}\right), \\
& \beta_{g}=k z_{g} \times d_{g}, \quad \beta_{d}=k z_{d} \times d_{d}, \\
& p p_{p}=\frac{\cos \left(\theta_{p}\right)}{n_{p}}, \quad p p_{g}=\frac{\cos \left(\theta_{g}\right)}{n_{g}}, \\
& p p_{d}=\frac{\cos \left(\theta_{d}\right)}{n_{d}}, \quad p p_{s}=\frac{\cos \left(\theta_{s}\right)}{n_{s}}, \\
& n_{g} \sin \left(\theta_{g}\right)=n_{p} \sin (\theta), \quad n_{d} \sin \left(\theta_{d}\right)=n_{p} \sin (\theta), \\
& n_{s} \sin \left(\theta_{s}\right)=n_{p} \sin (\theta), \\
& t H=\frac{2 p p_{p}}{\left(m_{11}+m_{12} \times p p_{s}\right) p p_{p}+\left(m_{21}+m_{22} \times p p_{s}\right)}, \\
& t E=\frac{n_{p}}{n_{s}} t H,
\end{aligned}
$$

where $n_{p}, n_{g}, n_{d}$, and $n_{s}$ are the refractive indices of the prism, gold film, dielectric film, and samples, $z$ is the distance from the prism surface, $d_{g}$ and $d_{d}$ are the thickness of the gold film and dielectric film, and $\theta$ and $E_{i}$ are the incident angle and electric field strength of the incident light, respectively. Then, the electric field strength in the gold film $E_{g}$, dielectric film $E_{d}$, and samples $E_{s}$ can be calculated using

$$
\begin{aligned}
& E_{g}=\sqrt{\left(E x_{g}\right)^{2}+\frac{n_{p}^{2} \times \sin (\theta)}{\left(n_{g}\right)^{4}}\left(H y_{g}\right)^{2}} \\
& E_{d}=\sqrt{\left(E x_{d}\right)^{2}+\frac{n_{p}{ }^{2} \times \sin \theta}{\left(n_{d}\right)^{4}}\left(H y_{d}\right)^{2}} \\
& E_{s}=E i \times \sqrt{\left[\left(\cos \theta_{s} \times t E\right)^{2}+\left(\frac{n_{p} \sin (\theta)}{n_{s}} \times t E\right)^{2}\right]} \\
& \times \sqrt{\exp \left(\frac{-4 \pi\left(z-d_{g}-d_{d}\right) \operatorname{Im}\left(n_{s} \times \cos \theta_{s}\right)}{\lambda}\right)} \text {. }
\end{aligned}
$$

In the multilayer system, the thicknesses of different layers are of importance for the sensor characteristics. So we discuss the influence of the thickness of layer on the figure of merit and accuracy of finding resonance position. The accuracy of finding resonance position is estimated using the combined sensitivity factor (CSF) [24] as

$$
\mathrm{CSF}=S \times \frac{R_{\max }-R_{\min }}{\text { FWHM }}
$$

where the $\theta$ is the incident angle, $R_{\max }$ and $R_{\min }$ are the maximum and minimum normalized reflectance, FWHM is the full width at half maximum, and $S$ is the sensitivity factor. The surface and bulk CSFs are calculated using the surface and bulk sensitivities, respectively.

We study the effect of the thickness change of the gold film firstly. The thickness of the silica film is fixed to be $1000 \mathrm{~nm}$. The figure of merit, surface CSF, and bulk CSF with the gold film of $40,45,50,55,60,65$, and $70 \mathrm{~nm}$ are calculated and shown in Figures 2(a), 2(b), and 2(c), respectively. It can be seen that all three performance indices do not respond to the gold film thickness monotonously. The figure of merit, which is the most important estimate function in the self-referenced measurement, gets the maximum value at the gold film of approximately $65 \mathrm{~nm}$. But the surface and bulk CSFs in the TM1 and TM2 modes all achieve the best values at the range of approximately $55 \mathrm{~nm}$ to $60 \mathrm{~nm}$. While the figure of merit at the gold film of $55 \mathrm{~nm}$ or $60 \mathrm{~nm}$ is a little lower than the value at the gold film of $65 \mathrm{~nm}$, the CSFs at the gold film of $65 \mathrm{~nm}$ are worse than the values at the gold film of $55 \mathrm{~nm}$ and $60 \mathrm{~nm}$ obviously. After trading off the cross sensitivity and the accuracy of finding the resonance position, we consider that the 55 or $60 \mathrm{~nm}$ should be the optimization thickness for the gold film in this structure. For further confirmation, the angular reflectance spectra at the gold film of 55 and $60 \mathrm{~nm}$ are calculated and shown in Figure 2(d). It can be seen that 


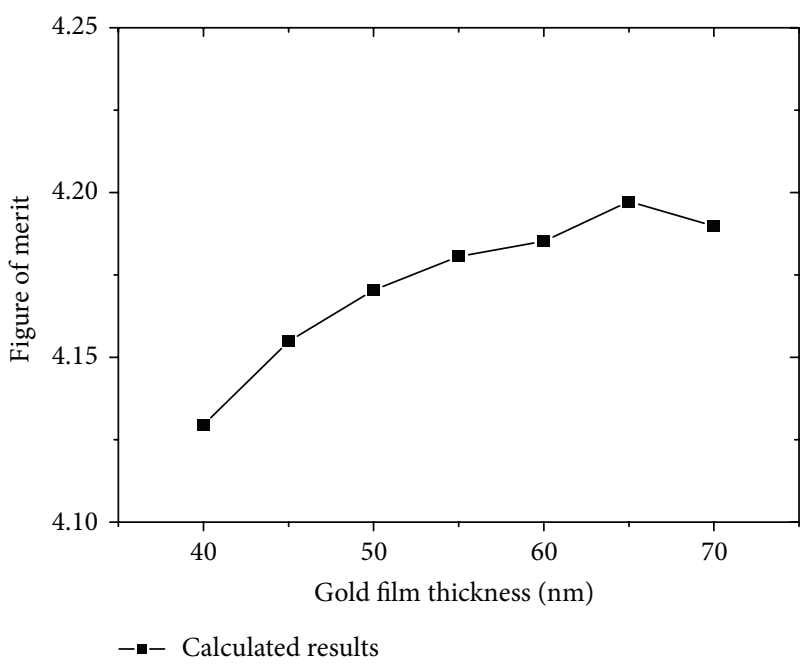

(a)

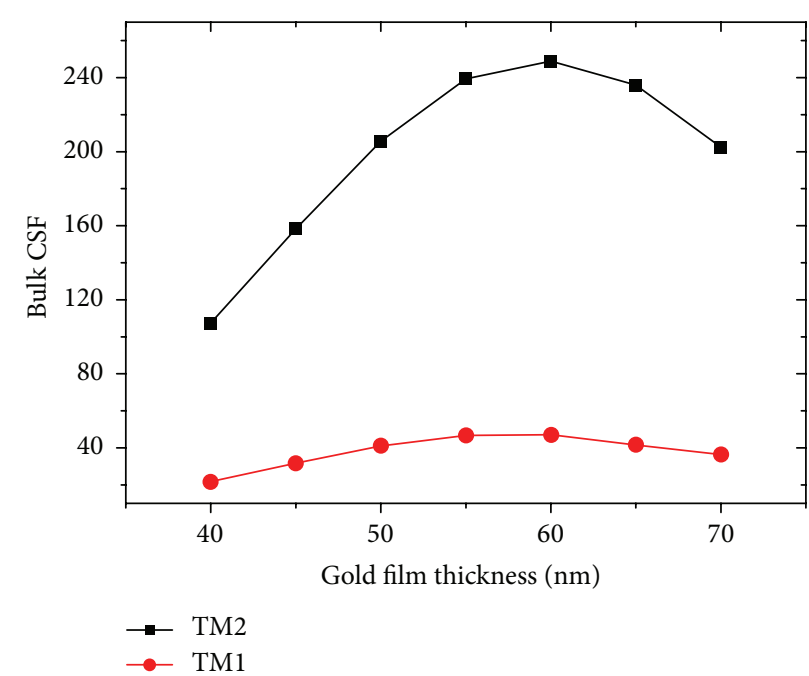

(c)

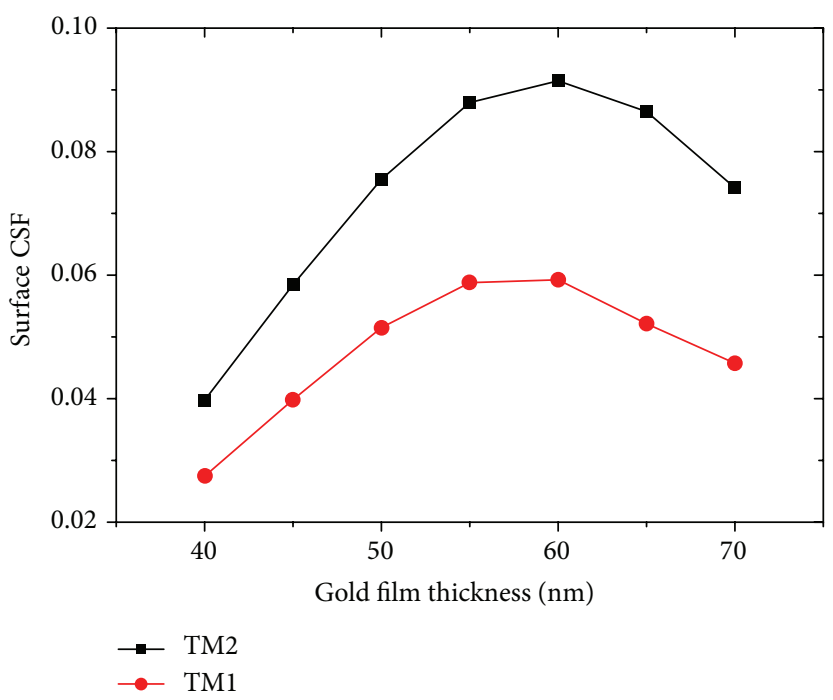

(b)

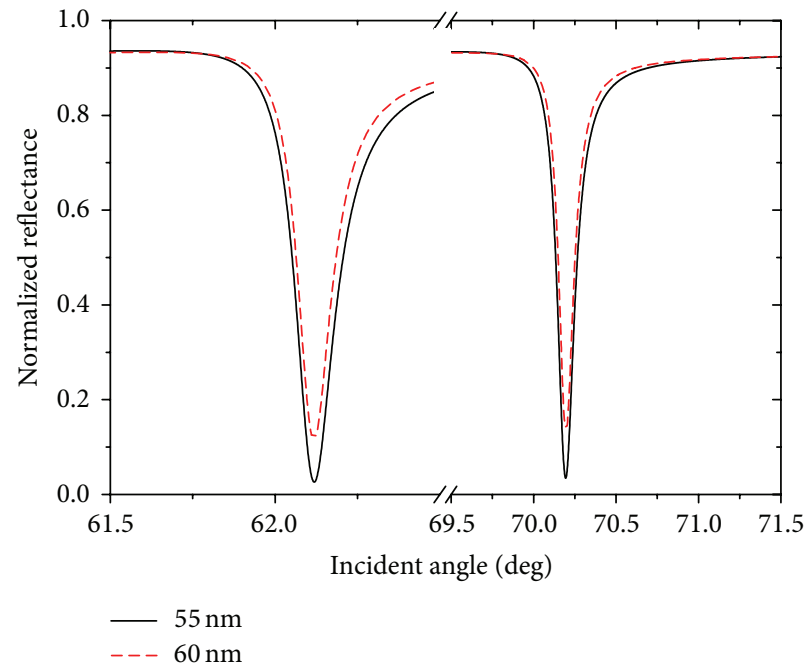

(d)

Figure 2: ((a), (b), and (c)) The figure of merit, surface combined sensitivity factor (CSF), and bulk CSF plotted against the gold film thickness. The silica films have the thickness of $1000 \mathrm{~nm}$. The refractive indices of the prism, gold, silica, and water are $1.515,0.133+3.654 i, 1.46$, and 1.33, respectively. The incident wavelength is $632.8 \mathrm{~nm}$. (d) The angular reflectance spectra with the gold film of $55 \mathrm{~nm}$ and $60 \mathrm{~nm}$.

there is better extinction ratio in the angular spectrum at the gold film of $55 \mathrm{~nm}$. This will be beneficial for the resonance angle measurement in the practical terms $[25,26]$. In this discussion, we choose the interval of $5 \mathrm{~nm}$ changing the gold film thickness. This is because there are always thickness errors in the vacuum film coating that have been studied in our previous work [27], so we think it should be unnecessary to use smaller interval. Considering these, we think the optimization thickness of the gold film is $55 \mathrm{~nm}$ in this structure.

The characteristics of the surface plasmon based optical sensors are usually corresponding to the electric field distributions of the evanescent field [28]. To illustrate the optimal gold film thickness of $55 \mathrm{~nm}$, the electric field strength distributions of the evanescent fields in TM1 and TM2 waveguide modes are calculated and shown in Figures 3(a) and 3(b) with different gold film thickness. It could be seen from Figure 3 that the electric field strengths are both lower when the gold film thickness is thinner or thicker than the optimal thickness. This may be because the incident light could not be efficiently coupled to the waveguide modes via the surface plasmons existing on the gold film surface due to the matching relations in the multilayer system. In summary, this PWR sensor has better characteristics when the gold film owns optimal thickness.

After optimizing the gold film thickness to be $55 \mathrm{~nm}$, we discuss the effect of the silica film thickness on the sensor characteristics. We should point out that the two waveguide modes in the silica layer should maintain a large RI range. 


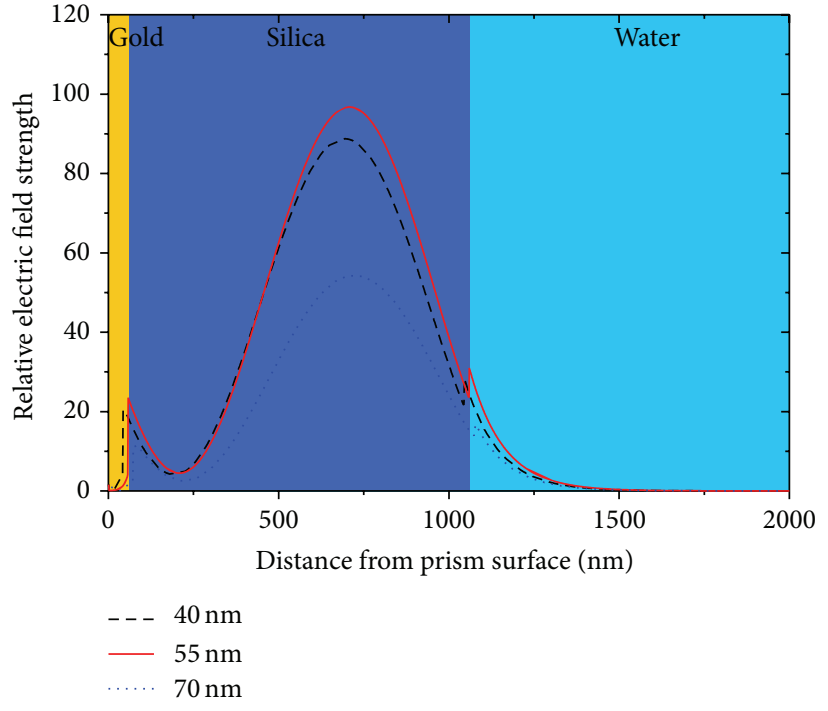

(a)

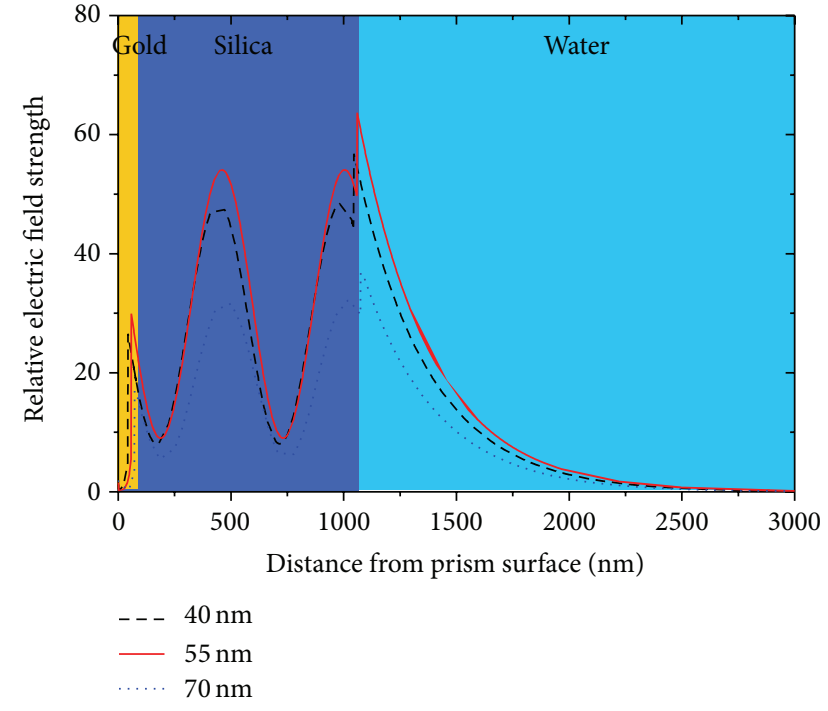

(b)

Figure 3: (a) The electric field strengths under resonance condition are plotted against the distance from the prism surface with the gold film thicknesses of 40,55, and $70 \mathrm{~nm}$ in TM1 waveguide mode and (b) in TM2 waveguide mode. The silica film has the thickness of $1000 \mathrm{~nm}$. The refractive indices of the prism, gold, silica, and water are $1.515,0.133+3.654 i, 1.46$, and 1.33 , respectively. The incident wavelength is $632.8 \mathrm{~nm}$.

Under room temperature, the water RI varies from 1.33 to 1.34 in the visible light wavelength region [18]. So we change the water RI from 1.33 to 1.34 to test the angular reflectance spectrum in this sensor. We calculate the angular reflectance spectra with the silica film of $900 \mathrm{~nm}, 950 \mathrm{~nm}$, and $1000 \mathrm{~nm}$ and show them in Figure 4(a). It can be seen that the angular reflectance spectra in TM2 mode cannot maintain good resonance curve in this range until the silica film thickness increases to $1000 \mathrm{~nm}$. Then we increase the silica film thickness and the bulk CSFs, surface CSFs, and figure of merit in the TM1 and TM2 modes are calculated and shown in Figures 4(b), 4(c), and 4(d), respectively. Different from the gold film, the silica film thickness increase has negative effect on all three performance indices. It may be because the electric fields in two modes are more limited in the silica film with increasing the silica film thickness. The electric field distributions of the evanescent fields existing in the water under resonance conditions in TM1 and TM2 waveguide modes are calculated as shown in Figures 5(a) and $5(\mathrm{~b})$, respectively. It can be seen from Figure 5 that the electric field strengths of evanescent fields in two waveguide modes both decrease with increasing the silica film thickness. In summary, the optimization silica film thickness is exactly the critical thickness, where the two waveguide modes just can maintain the good resonance curves. So the optimization thickness of the gold and silica films in this structure is 55 and $1000 \mathrm{~nm}$, respectively.

\section{Discussion}

There are six parameters (e.g., prism RI, metal film thickness and RI, dielectric layer thickness and RI, and incident wavelength) in this self-referenced PWR sensor so there will be more opportunities for minimizing the cross sensitivity and improving the accuracy of finding the resonance position. It has been pointed out that the performance of conventional PWR sensor can be improved by lowering the RI of dielectric layer [29]. Then we employ magnesium fluoride $\left(\mathrm{MgF}_{2}\right)$ with RI of 1.38, which has also been used in the PWR sensor [12], instead of the silica with the RI of 1.46 as the dielectric layer. We have known that the optimization dielectric layer thickness is the critical thickness. The gold film of $55 \mathrm{~nm}$ is also employed as the metal film. To confirm the critical thickness of $\mathrm{MgF}_{2}$ layer, the angular reflectance spectra with the $\mathrm{MgF}_{2}$ layer thickness of $1600 \mathrm{~nm}$ and $1650 \mathrm{~nm}$ measuring the RI of 1.33 and 1.34 are calculated and shown in Figure 6(a). It can be seen from Figure 6(a) that the critical thickness of $\mathrm{MgF}_{2}$ layer for the self-referenced measurement is $1650 \mathrm{~nm}$. The electric field distribution with $\mathrm{MgF}_{2}$ layer thickness of $1650 \mathrm{~nm}$ is calculated and shown in Figure 6(b). It can be seen from Figure 6(b) that the electric field distribution in this system is similar to the gold-silica systems shown in Figure 1. To compare the sensing characteristics of two structures, the angular reflectance spectra with the $\mathrm{MgF}_{2}$ film of $1650 \mathrm{~nm}$ and silica film of $1000 \mathrm{~nm}$ are calculated and shown in Figure 7(a). Then we calculate the electric field distributions of the evanescent fields in the water in the TM1 and TM2 waveguide modes in both sensing structures and show them in Figure 7(b).

It can be seen that the resonance curves in the gold$\mathrm{MgF}_{2}$ structure are sharper than the gold-silica structure. The surface and bulk CSFs in the TM2, TM1 modes are 0.13 and 528, 0.11 and 136 in the gold- $\mathrm{MgF}_{2}$ structure, which are better than the 0.088 and $239,0.059$ and 47 in the goldsilica structure. The figure of merit, essential performance index in the self-referenced measurement, is 5.31 in the gold$\mathrm{MgF}_{2}$ structure, which is better than the gold-silica film 


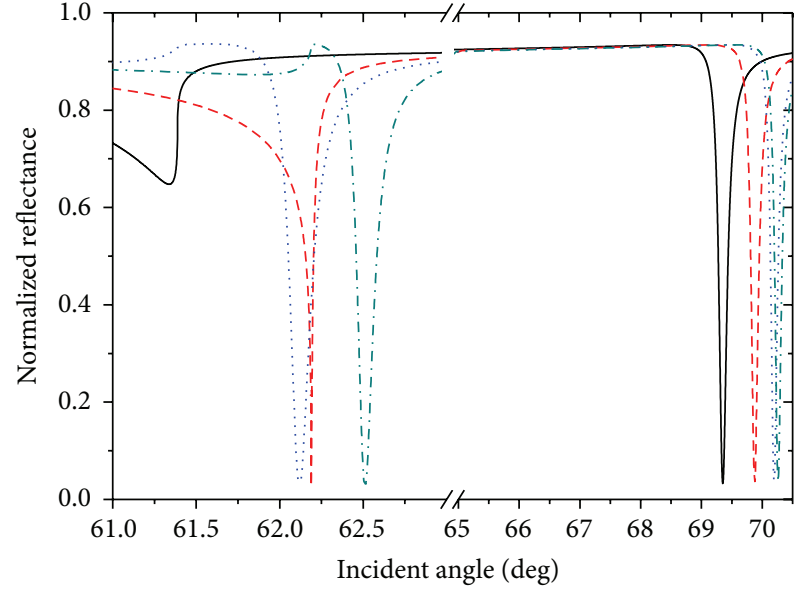

— $900 \mathrm{~nm}$ with $1.33 \quad \ldots \ldots 1000 \mathrm{~nm}$ with 1.33

(a)

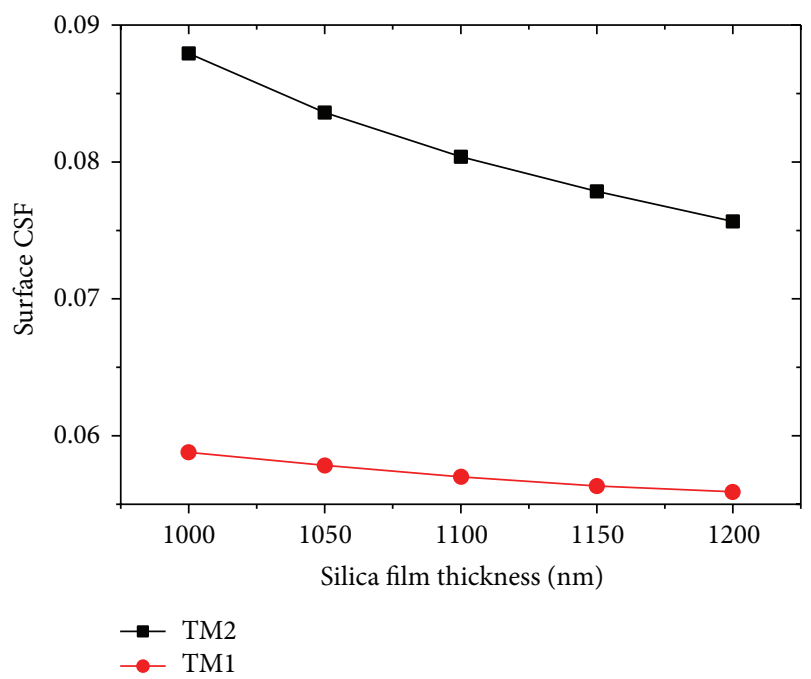

(c)

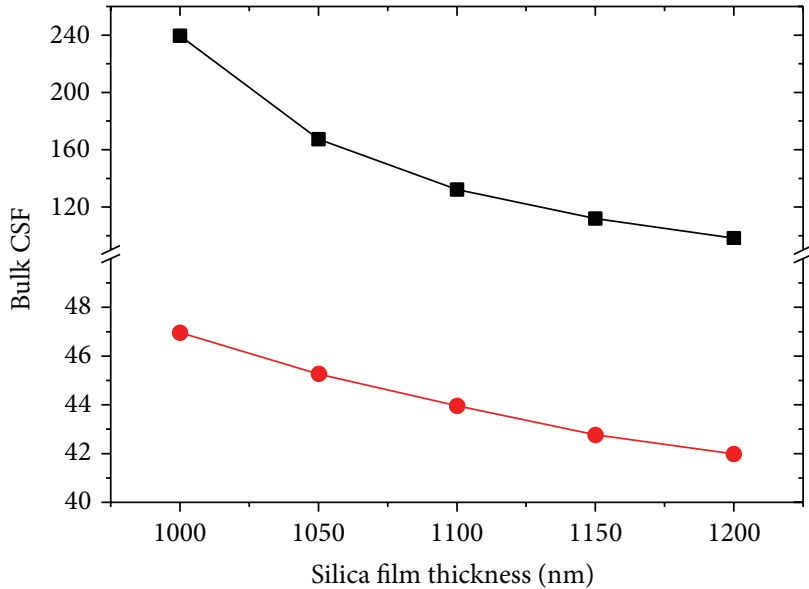

TM2

TM1

(b)

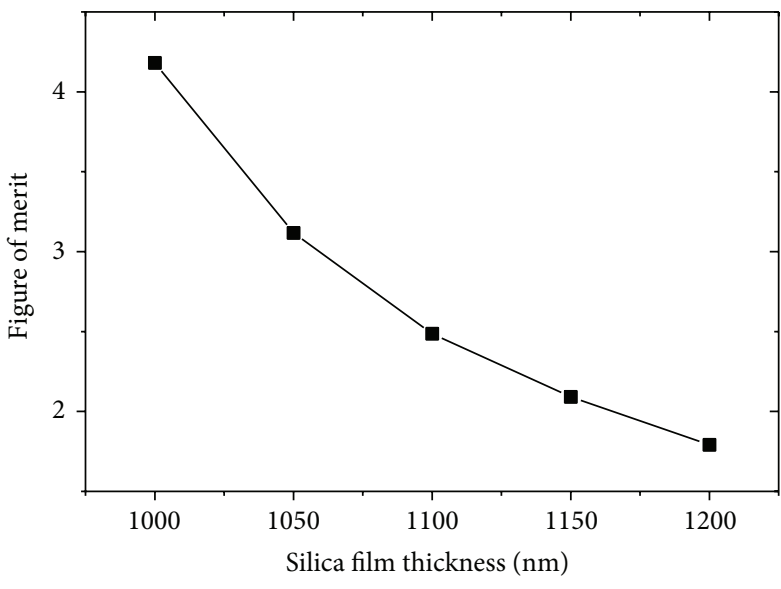

- Calculated results

(d)

FIGURE 4: (a) The angular reflectance spectra with different silica film thickness measuring the samples with different refractive indices. ((b), (c), and (d)) The bulk combined sensitivity factor (CSF), surface CSF, and figure of merit plotted against the silica film thickness. The gold films have the thickness of $55 \mathrm{~nm}$. The refractive indices of the prism, gold, silica, and water are 1.515, $0.133+3.654 i, 1.46$, and 1.33 , respectively. The incident wavelength is $632.8 \mathrm{~nm}$.

structure with the value of 4.18 and the reported values of 1.4 [7], 1.46 [8], 5.0 [9], 3.8 [10], and 4.7 [14]. The improvement by the employment of $\mathrm{MgF}_{2}$ can be owing to the enhanced electric field shown in Figure 7(b). It can be seen that the electric fields in the analyte are both enhanced in the TM1 and TM2 modes. There is an additional advantage of the gold$\mathrm{MgF}_{2}$ structure; the resonance angle difference between two waveguide modes is less than 3 degrees, which is comparable with the reported long range SPR structure [9] but much less than the reported self-referenced PWR using TM and TE modes [14]. This will lead to easier detection module arrangement in the practical terms. This discussion shows this self-referenced PWR sensing structure owns good potential to be improved owing to the multisensor parameters.

\section{Conclusion}

In this paper, we report a PWR sensing structure supporting two waveguide modes for self-referenced measurement. By simply increasing the dielectric layer thickness, the sensing structure supporting two waveguide modes with different penetration depths in the analyte can be constructed. We have optimized the structure in terms of high accuracy and low cross sensitivity for both modes. The optimized dual 


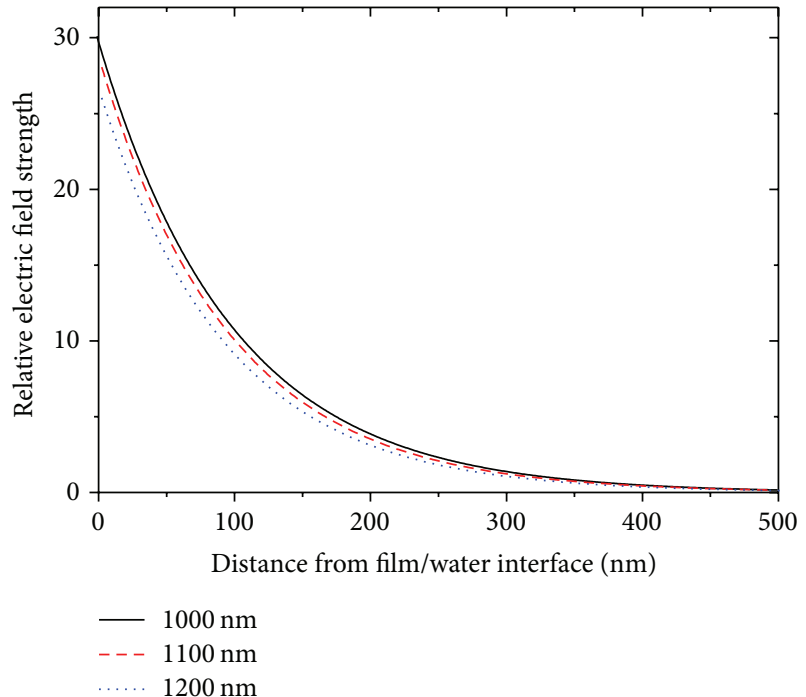

(a)

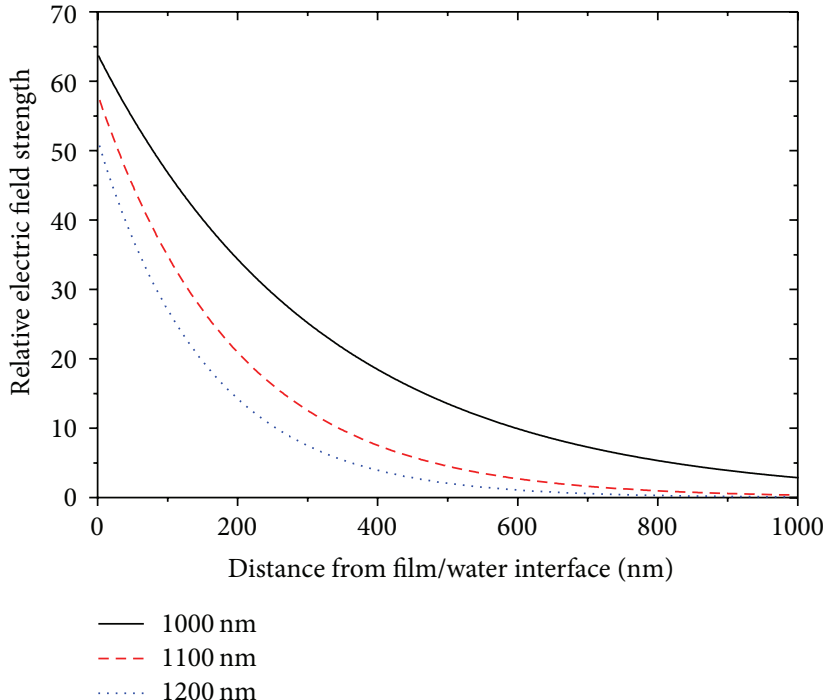

(b)

FIGURE 5: (a) The electric field strengths under resonance condition are plotted against the distance from the film/water interface with the silica film thickness of $1000 \mathrm{~nm}, 1100 \mathrm{~nm}$, and $1200 \mathrm{~nm}$ in TM1 waveguide mode and (b) in TM2 waveguide mode. The gold film has the thickness of $55 \mathrm{~nm}$. The refractive indices of the prism, gold, silica, and water are 1.515, $0.133+3.654 i, 1.46$, and 1.33 , respectively. The incident wavelength is $632.8 \mathrm{~nm}$.

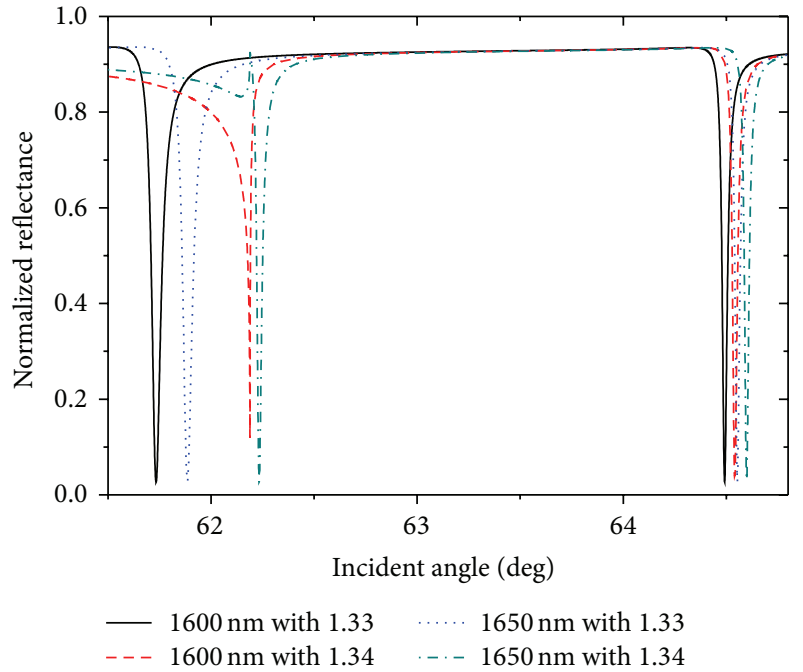

(a)

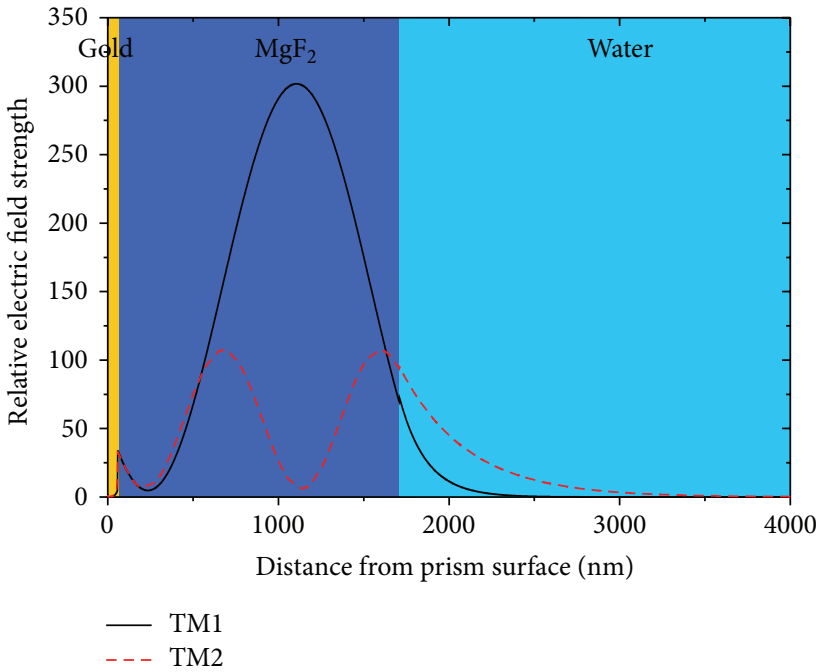

(b)

FiguRE 6: (a) The angular reflectance spectra with the $\mathrm{MgF}_{2}$ film thickness of $1600 \mathrm{~nm}$ and $1650 \mathrm{~nm}$ measuring the samples with different refractive indices. (b) The electric field strengths in different waveguide modes under resonance condition are plotted against the distance from the prism surface. The gold film has the thickness of $55 \mathrm{~nm}$. The refractive indices of the prism, gold, silica, and water are $1.515,0.133+$ $3.654 i, 1.46$, and 1.33 , respectively. The incident wavelength is $632.8 \mathrm{~nm}$.

mode chip with gold-silica structure owns a figure of merit of 4.18. After using the gold- $\mathrm{MgF}_{2}$ structure, the figure of merit is improved to 5.31. The detection operation can be accomplished with only the TM polarized incident light using the attenuated total reflection manner, so it provides a selfreferenced approach with good compatibility for the common SPR and PWR sensors. Further work is required for experimental demonstration of the feasibility of this structure. 


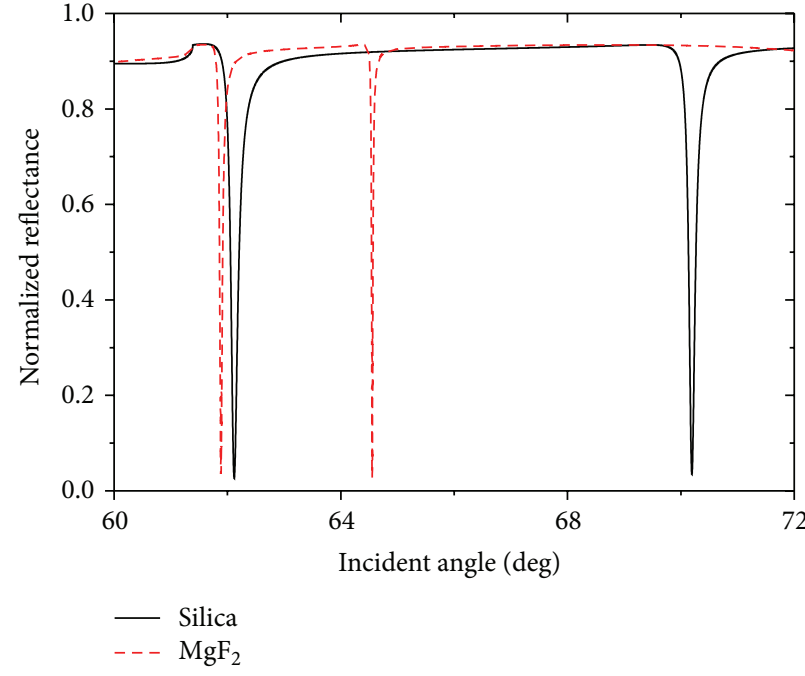

(a)

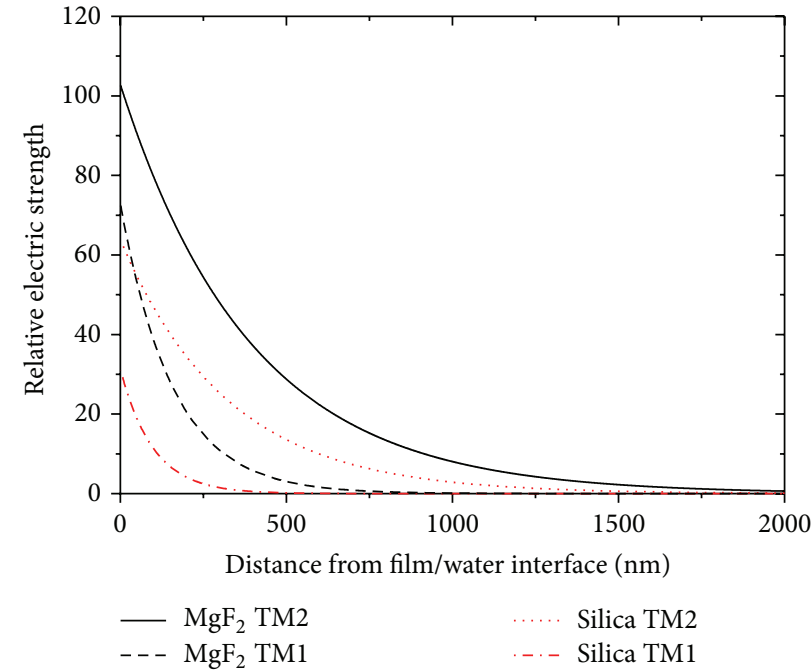

(b)

FiguRE 7: (a) The comparison of angular reflectance spectra with silica film and $\mathrm{MgF}_{2}$ film as the dielectric layers. The gold film, silica film, and $\mathrm{MgF}_{2}$ film have the thickness of $55 \mathrm{~nm}, 1000 \mathrm{~nm}$, and $1650 \mathrm{~nm}$. The refractive indices of the prism, gold, silica, $\mathrm{MgF}_{2}$, and water are 1.515 , $0.133+3.654 i, 1.46,1.38$, and 1.33 , respectively. The incident wavelength is $632.8 \mathrm{~nm}$. (b) The electric field strengths in different waveguide modes under resonance condition are plotted against the distance from the film/water interface.

\section{Conflict of Interests}

The authors declare that there is no conflict of interests regarding the publication of this paper.

\section{Acknowledgments}

This research was made possible with the financial support from NSFC China (61275188, 61378089, and 61361160416), the 863 project, China, and the Technology Development Program of Shenzhen City.

\section{References}

[1] J. Homola, S. S. Yee, and G. Gauglitz, "Surface plasmon resonance sensors: review," Sensors and Actuators, B: Chemical, vol. 54, no. 1, pp. 3-15, 1999.

[2] J. Homola, "Surface plasmon resonance sensors for detection of chemical and biological species," Chemical Reviews, vol. 108, no. 2, pp. 462-493, 2008.

[3] B. D. Gupta and R. K. Verma, "Surface plasmon resonancebased fiber optic sensors: principle, probe designs, and some applications," Journal of Sensors, vol. 2009, Article ID 979761, 12 pages, 2009.

[4] L. Liu, S. Ma, Y. Ji et al., "A two-dimensional polarization interferometry based parallel scan angular surface plasmon resonance biosensor," Review of Scientific Instruments, vol. 82, no. 2, Article ID 023109, 2011.

[5] J. T. Hastings, "Optimizing surface-plasmon resonance sensors for limit of detection based on a Cramer-Rao bound," IEEE Sensors Journal, vol. 8, no. 2, pp. 170-175, 2008.

[6] Z. Liu, L. Liu, X. Wang et al., "Polarization-interferometrybased wavelength-interrogation surface plasmon resonance imager for analysis of microarrays," Journal of Biomedical Optics, vol. 17, no. 3, Article ID 036002, 2012.
[7] R. Slavík, J. Homola, and H. Vaisocherová, "Advanced biosensing using simultaneous excitation of short and long range surface plasmons," Measurement Science and Technology, vol. 17, no. 4, pp. 932-938, 2006.

[8] J. T. Hastings, J. Guo, P. D. Keathley et al., "Optimal self-referenced sensing using long- and short-range surface plasmons," Optics Express, vol. 15, no. 26, pp. 17661-17672, 2007.

[9] J. Guo, P. D. Keathley, and J. T. Hastings, "Dual-mode surfaceplasmon-resonance sensors using angular interrogation," Optics Letters, vol. 33, no. 5, pp. 512-514, 2008.

[10] G. Dyankov, M. Zekriti, and M. Bousmina, "Dual-mode surface-plasmon sensor based on bimetallic film," Applied Optics, vol. 51, no. 13, pp. 2451-2456, 2012.

[11] Z. Salamon, H. A. Macleod, and G. Tollin, "Coupled plasmonwaveguide resonators: a new spectroscopic tool for probing proteolipid film structure and properties," Biophysical Journal, vol. 73, no. 5, pp. 2791-2797, 1997.

[12] H. Shi, Z. Y. Liu, X. X. Wang et al., "A symmetrical optical waveguide based surface plasmon resonance biosensing system," Sensors and Actuators B: Chemical, vol. 185, pp. 91-96, 2013.

[13] F. Bahrami, M. Maisonneuve, M. Meunier, J. Stewart Aitchison, and M. Mojahedi, "An improved refractive index sensor based on genetic optimization of plasmon waveguide resonance," Optics Express, vol. 21, no. 18, pp. 20863-20872, 2013.

[14] F. Bahrami, M. Maisonneuve, M. Meunier, J. S. Aitchison, and M. Mojahedi, "Self-referenced spectroscopy using plasmon waveguide resonance biosensor," Biomedical Optics Express, vol. 5, no. 8, pp. 2481-2487, 2014.

[15] Y. Fan, K. Hotta, A. Yamaguchi, and N. Teramae, "Enhanced fluorescence in a nanoporous waveguide and its quantitative analysis," Optics Express, vol. 20, no. 12, pp. 12850-12859, 2012.

[16] S. Szunerits and R. Boukherroub, "Preparation and characterization of thin films of $\mathrm{SiO}_{x}$ on gold substrates for surface 
plasmon resonance studies," Langmuir, vol. 22, no. 4, pp. 1660$1663,2006$.

[17] H. Imai, M. Yasumori, H. Hirashima, K. Awazu, and H. Onuki, "Significant densification of sol-gel derived amorphous silica films by vacuum ultraviolet irradiation," Journal of Applied Physics, vol. 79, no. 11, pp. 8304-8309, 1996.

[18] P. Schiebener, J. Straub, J. M. H. L. Sengers, and J. S. Gallagher, "Refractive index of water and steam as function of wavelength, temperature and density," Journal of Physical and Chemical Reference Data, vol. 19, no. 3, pp. 677-717, 1990.

[19] S. K. Ozdemir and G. Turhan-Sayan, "Temperature effects on surface plasmon resonance: design considerations for an optical temperature sensor," Journal of Lightwave Technology, vol. 21, no. 3, pp. 805-814, 2003.

[20] O. S. Heavens, Optical Properties of Thin Films, Dover, New York, NY, USA, 1955.

[21] M. Born and E. Wolf, Principles of Optics: Electromagnetic Theory of Propagation, Interference and Diffraction of Light, CUP Archive, 1999.

[22] W. N. Hansen, "Electric fields produced by the propagation of plane coherent electromagnetic radiation in a stratified medium," Journal of the Optical Society of America, vol. 58, no. 3, pp. 380-390, 1968.

[23] P. K. Tien and R. Ulrich, "Theory of prism-film coupler and thin-film light guides," Journal of the Optical Society of America, vol. 60, no. 10, pp. 1325-1337, 1970.

[24] A. Abbas, M. J. Linman, and Q. Cheng, "Sensitivity comparison of surface plasmon resonance and plasmon-waveguide resonance biosensors," Sensors and Actuators B: Chemical, vol. 156, no. 1, pp. 169-175, 2011.

[25] P. F. Zhang, L. Liu, Y. H. He et al., "Non-scan and realtime multichannel angular surface plasmon resonance imaging method," Applied Optics, vol. 53, no. 26, pp. 6037-6042, 2014.

[26] Y. F. Zhou, P. F. Zhang, Y. H. He et al., "Plasmon waveguide resonance sensor using an $\mathrm{Au}-\mathrm{MgF}_{2}$ structure," Applied Optics, vol. 53, no. 28, pp. 6344-6350, 2014.

[27] L. Liu, X. Chen, Z. Liu et al., "Polarization interference interrogation of angular surface plasmon resonance sensors with wide metal film thickness tolerance," Sensors and Actuators, B: Chemical, vol. 173, pp. 218-224, 2012.

[28] A. Lahav, M. Auslander, and I. Abdulhalim, "Sensitivity enhancement of guided-wave surface-plasmon resonance sensors," Optics Letters, vol. 33, no. 21, pp. 2539-2541, 2008.

[29] N. Skivesen, R. Horvath, and H. C. Pedersen, "Optimization of metal-clad waveguide sensors," Sensors and Actuators B: Chemical, vol. 106, no. 2, pp. 668-676, 2005. 

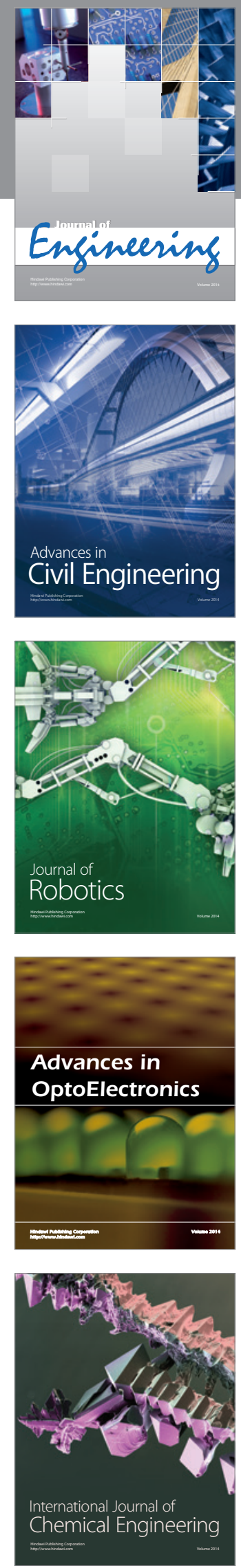

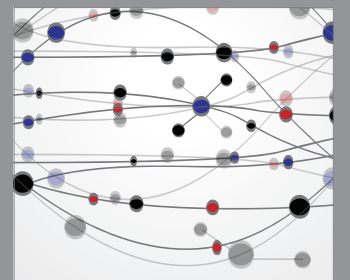

The Scientific World Journal
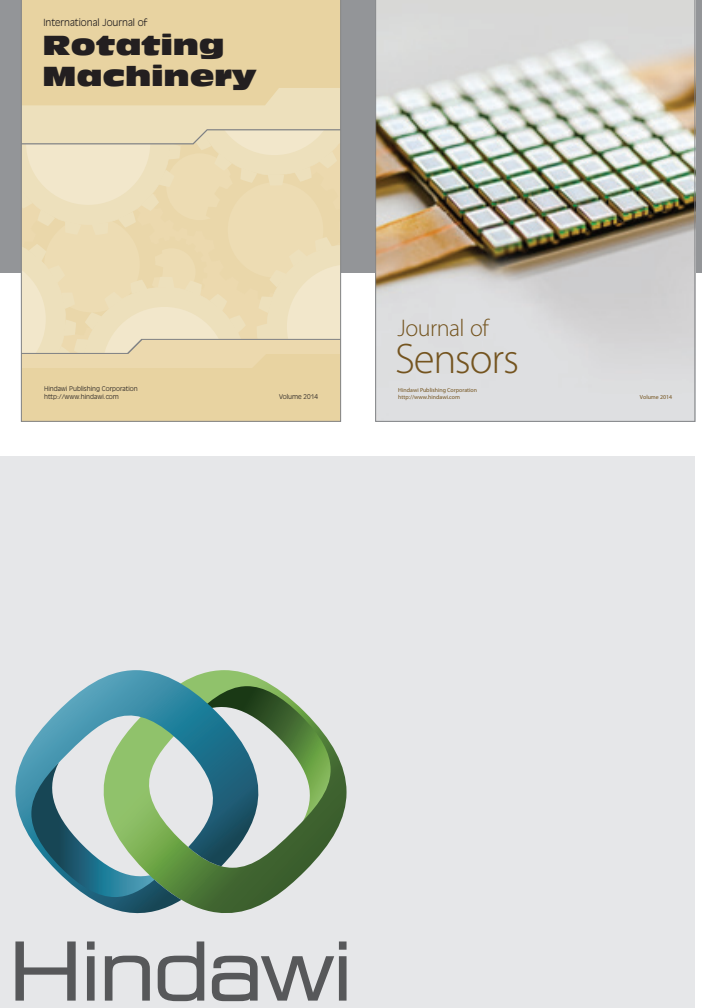

Submit your manuscripts at http://www.hindawi.com
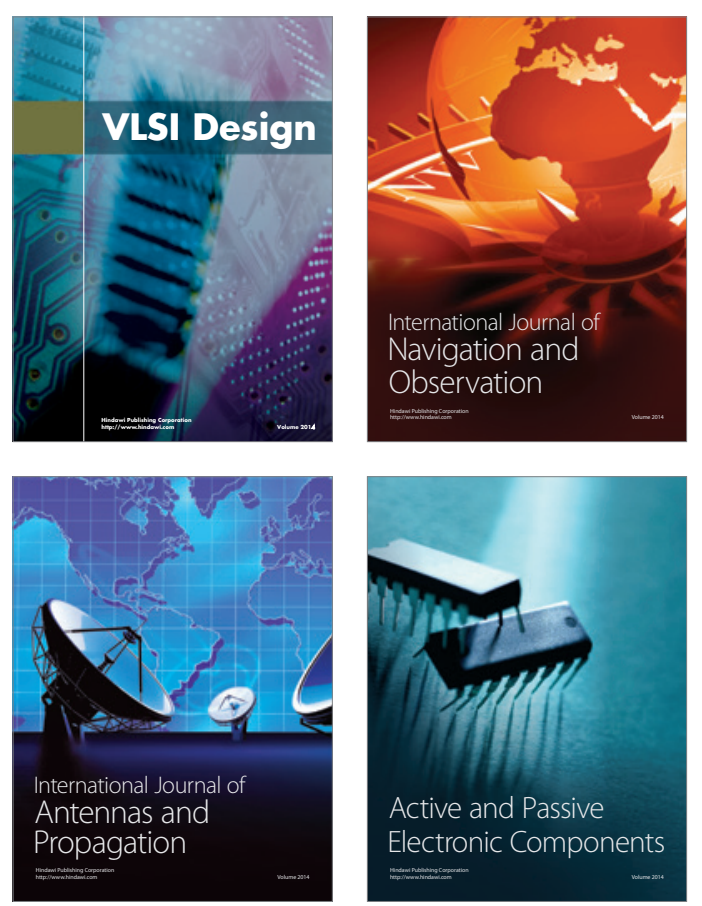
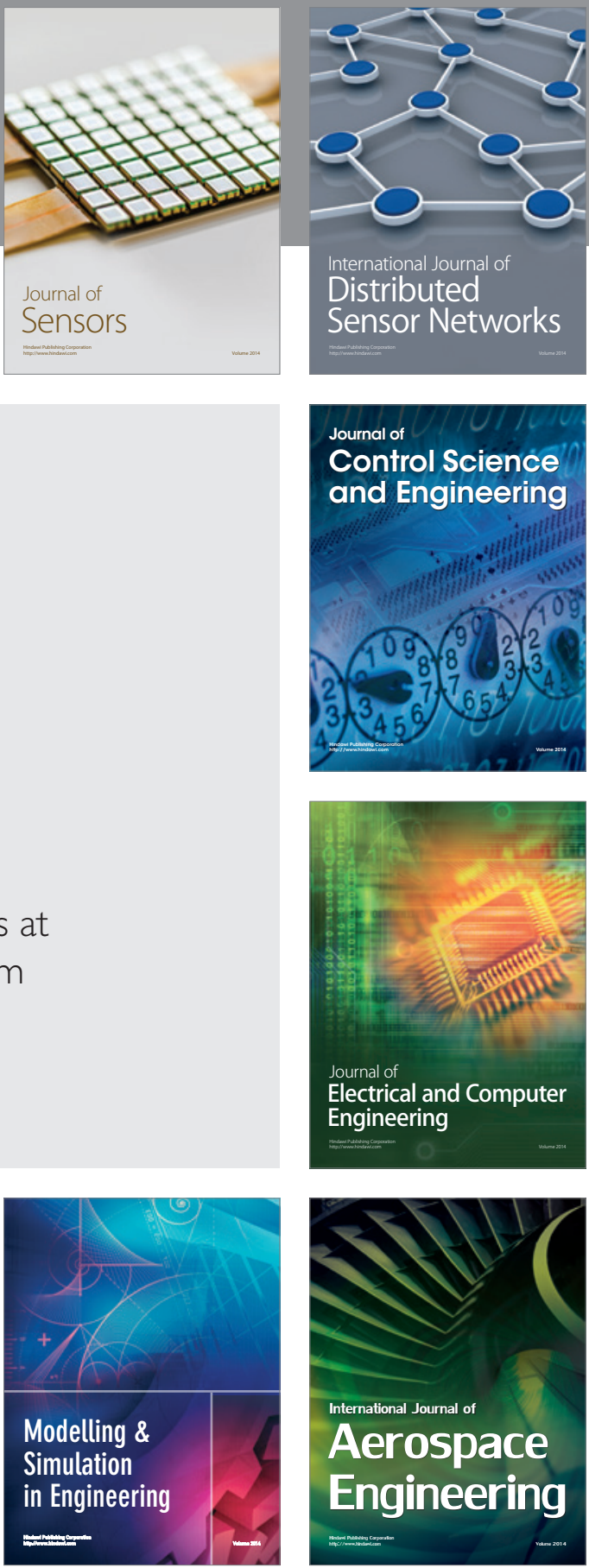

Journal of

Control Science

and Engineering
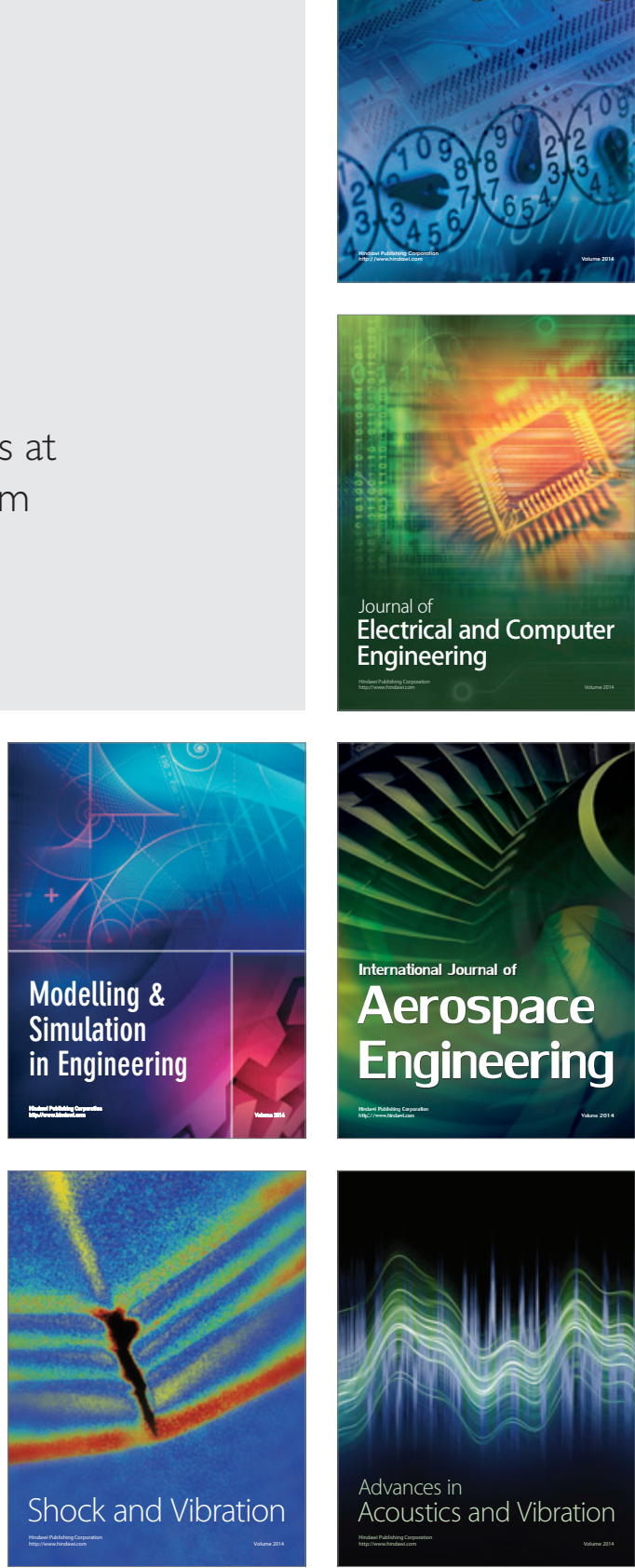